\title{
Role of Alanine Aminotransferase in determining the biliary etiology in Acute Pancreatitis
}

\author{
Shailesh Simkhada, Parashuram Mishra \\ Department of Surgery, Institute of Medicine, Maharajgunj Campus \\ Tribhuvan University Teaching Hospital \\ Kathmandu, Nepal
}

Correspondence: Shailesh Simkhada, MS Resident, Department of Surgery, Institute of Medicine, Tribhuvan University Teaching Hospital, Kathmandu Nepal

Email: simka.smart@gmail.com

\begin{abstract}
Background: Acute pancreatitis (AP) is a common surgical presentation and a leading cause of morbidity and mortality worldwide. Gallstones and excessive alcohol consumption are the most frequent causes of AP. Aim of this study was to investigate the predictive value of raised ALT (Alanine aminotransferase) in determining biliary etiology in patients presenting with acute pancreatitis.
\end{abstract}

Materials and Methods: All patients admitted in the surgical ward of Tribhuvan University Teaching Hospital with the diagnosis of AP were studied prospectively over a period of 1 year between 2014and 2015. Peak ALT within $48 \mathrm{~h}$ of presentation was recorded. Etiology was determined on the basis of history, Abdominal Ultrasound (AUS) and other relevant investigations.

Results: A total of 80 patients of AP were included in the study. Among them, 46 (57.5\%) patients had biliary pancreatitis, $18(22.5 \%)$ had alcoholic pancreatitis, $4(5 \%)$ patients had drug induced pancreatitis and $12(15 \%)$ patients had idiopathic pancreatitis. 46(57.5\%) were male and 34(42.5\%) were female. Mean ALT for biliary pancreatitis was $212.5 \mathrm{U} / \mathrm{L}$, for alcoholic was $58 \mathrm{U} / \mathrm{L}$, for drug induced was $71 \mathrm{U} / \mathrm{L}$ and for idiopathic was $43 \mathrm{U} / \mathrm{L}$. When compared between biliary and non-biliary pancreatitis, it was statistically significant $(p$ value $<0.00)$. Biliary was the most common etiology followed by alcoholic. The sensitivity, specificity, positive predictive value(PPV) and negative predictive value(NPV) of alanine aminotransferase with cut off of $100 \mathrm{U} / \mathrm{L}$ for biliary pancreatitis was $65 \%, 94 \%, 91.5 \%$ and $72.8 \%$ respectively.

Conclusion: An elevated ALT strongly supports a diagnosis of gallstones in Acute Pancreatitis.

Keywords: Acute Pancreatitis, Revised Atlanta classification, Alanine aminotransferase 